\title{
Partonic EoS in High-Energy Nuclear Collisions at RHIC
}

\author{
$\mathrm{Nu} \mathrm{Xu}$ \\ Nuclear Science Division \\ Lawrence Berkeley National Laboratory, Berkeley, CA 94720, USA
}

\begin{abstract}
We discuss the recent results on equation of state for partonic matter created at RHIC. Issues of partonic collectivity for multi-strange hadrons and $J / \psi$ from $A u+A u$ collisions at $\sqrt{s_{\mathrm{NN}}}=200 \mathrm{GeV}$ are the focus of this paper.

PACS numbers: 25.75.Dw, 13.20.Fc, 13.25.Ft, 24.85.+p
\end{abstract}

\section{Introduction}

The purpose of the heavy ion program at Brookhaven National Laboratory is to probe strongly interacting matter under extreme conditions, i.e. at high densities and temperatures. Naturally the search for the existence of a new form of matter - the quark-gluon plasma (QGP) - is the experimental focus of the program.

Lattice QCD calculations [1, 2] predict a transition or fast cross-over between QGP and the hadronic state at $T_{c} \approx 150-180 \mathrm{MeV}$ with vanishing baryon density. Including finite baryon density does not affect the general properties of the transition in Lattice QCD [3]. The energy density at the transition point is determined to be $\epsilon_{c} \approx 0.7-1.0$ $\mathrm{GeV} / \mathrm{fm}^{3}$. Under the same condition, chiral symmetry restoration also happens [1]. Therefore experimentally one would search for signatures of both QGP formation and in-medium effects of hadron properties.

In high-energy nuclear collisions, the term flow has two important aspects: (i) collectivity of produced hadrons and (ii) the local thermalization among these hadrons [4]. As long as there are interactions among constituents, collectivity of the matter will be developed provided that the distribution of matter density is not homogeneous. When the interactions last long enough the system will approach local equilibrium and hence develops hydrodynamic type flow. At the early stage of high-energy nuclear collision, both the matter density and its gradient are large, therefore we expect the development of partonic collectivity - the collective motion of partons. The issues of thermalization can be addressed by studying heavy-flavor $(c-, b-$ quarks) collectivity [5], because the collisions that generate the collective motion for heavy-quarks will likely lead to thermalization among the light quarks $(u-, d-, s-$ quarks) and gluons. It is 
important to note that collectivity is cumulative through the expansion phase and is not affected by the details of the hadronization process $\neq$.

At RHIC, the observation of 'jet-quenching' [8] and strong collectivity for all identified hadrons [9] has provided the evidence of hot and dense medium created in $A u+A u$ collisions. These experimental effects are most likely caused by the interactions among the partons $[9,10,11]$. This report will focus on the moset recent results on the issues of partonic equation of state, especially the heavy flavor collectivity results.

\section{Sysytematics of Hadron Slope Parameters}

At RHIC, the mid-rapidity invariant momentum distributions have been measured for pions, Kaons, protons [12], $\phi[13], \Lambda, \Xi, \Omega[14,15]$ and $J / \psi[18]$ from $\sqrt{s_{\mathrm{NN}}}=200 \mathrm{GeV}$ $A u+A u$ collisions. While pion spectra show a $p_{T}$-power-law shape, most of the hadron spectra are $m_{T}$-exponential, especially the strange hadrons like $K, \phi, \Xi, \Omega$ and $J / \psi$. In order to characterize the transverse motion, it is instructive to analyze the systematic mass dependent of the slope parameters extracted from the exponential fit:

$$
f_{\text {exp }}=A \cdot e^{-m_{T} / T}
$$

where $A$ and $T$ are the normalization constant and inverse slope parameter, respectively. The $m_{T}$ is defined as $m_{T}=\sqrt{p_{T}^{2}+m^{2}}$. The slope parameters from the central $A u+A u$ collisions at RHIC are shown in Figure 1. For comparison, also shown in the figure is the slope parameters from $\mathrm{Pb}+\mathrm{Pb}$ collisions at SPS top energy $[16,17,19,20]$.

As experected, the slope parameters increase as the hadrom mass. However, the mass dependence appears to have two branches:

(i) For copiously produced hadrons, $\pi, K, p$ and $d$. Here the mass dependent is strongly indicating an explosive expansion occurred in such heavy-ion collisions. A much stronger collective expansion is observed for collisions at RHIC is observed when compared with that from SPS energy.

(ii) Multi-strange hadrons $\phi, \Lambda, \Xi, \Omega$ and heavy flavor $J / \psi$. Here the slope parameter dependent on hadron mass is weaker compared with other lighter hadrons. At the SPS energy, on the other hand, there is no clear mass dependent, see the hatched band in Fig. 1. The finite values of the slope parameters at the SPS energy may reflect the string tension at hadronization [11].

As discussed in Ref. [20, 21], mult-strange and heavy flaovor hadrons do not participate in hadronic rescatterings and the collective motion can only be developed at the pre-hadronic stage, i.e. partonic stage for these hadrons. The clear mass dependent of the slope parameters in $A u+A u$ collisions at RHIC therefore provide a strong evidence for the partonic collectivity at RHIC.

$\ddagger$ The examples of such process can be found in hydrodynamic calculations[6] and a hadronization process via coalescence[7]. 


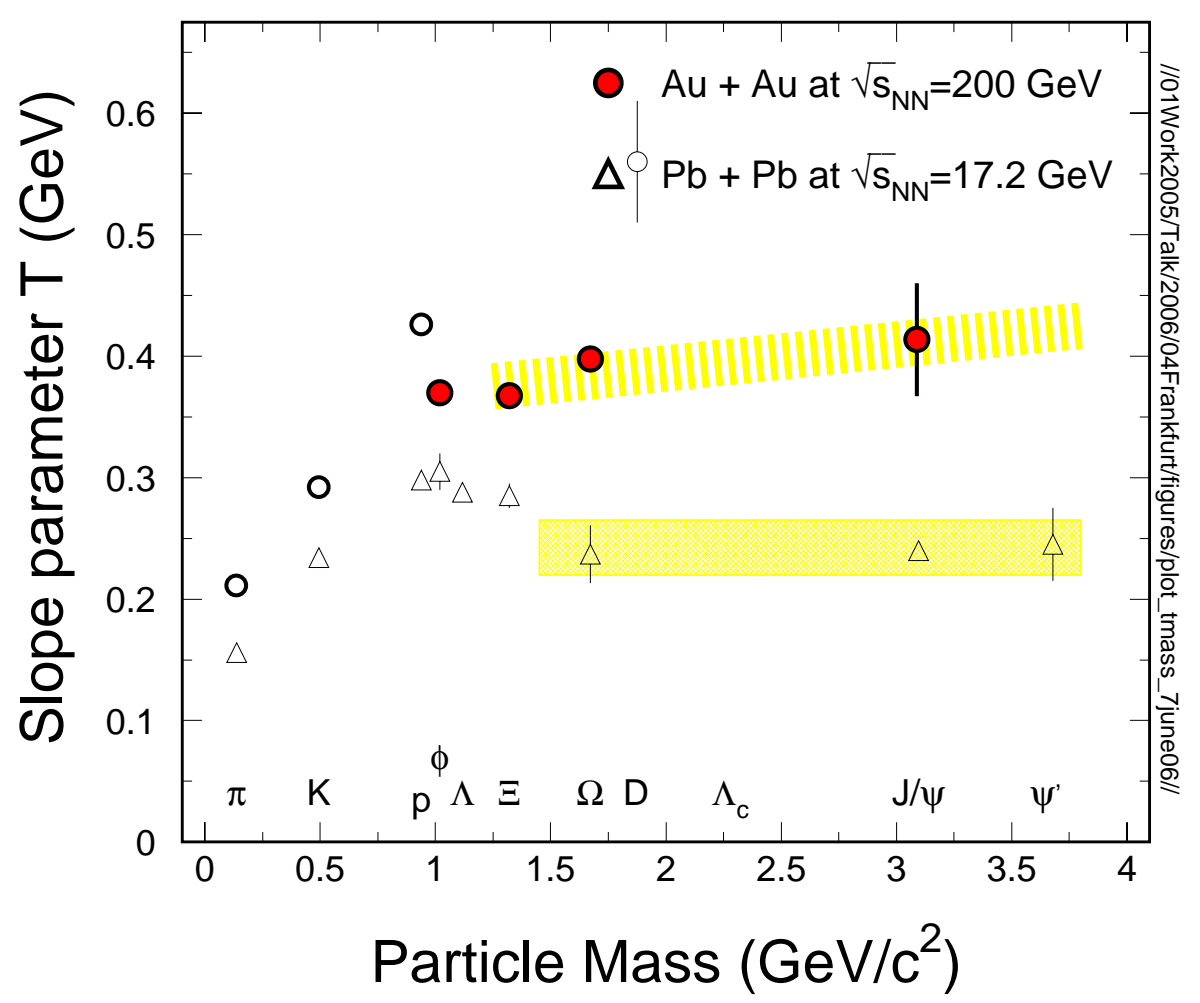

Figure 1. Mid-rapidity hadron alope parameters, as a function of mass, from central $P b+P b$ collisions at SPS at $\sqrt{s_{\mathrm{NN}}}=17.2 \mathrm{GeV}$ (triangles) and central Au+Au collisions at $\sqrt{s_{\mathrm{NN}}}=200 \mathrm{GeV}$ (circles).

From the hydrodynamics motivated fits $[9,11,22]$, at the most central collisions, the freeze-out parameters are $\left(T_{f o},\left\langle\beta_{T}\right\rangle\right)=(0.1 \pm 0.02(\mathrm{GeV}), 0.6 \pm 0.05(\mathrm{c}))$ and $(0.165 \pm 0.05$ $(\mathrm{GeV}), \geq 0.2(\mathrm{c}))$ for $\pi, K$ and $p$ and multi-hadron $\phi$ and $\Omega$, respectively. Since there are still large uncertainties in the determination of pre-equilibrium contribution to the extracted velocity parameters, we only have the lower limit for the partonic veloccity $\left\langle\beta_{T}\right\rangle_{p} \leq 0.2 \mathrm{c}$. However, these results are also consistent with the approach [19] where we assume both thermal motion and collective motion contribute to the measured slope parameter $T$ :

$$
T=T_{\text {thermal }}+A \cdot \text { mass } \cdot\left\langle\beta_{T}\right\rangle^{2} .
$$

It is interesting to note that the freeze-out temperature parameter for multi-strange and heavy flaovr hadrons are almost identical to the chemical freeze-out temperature $[11,9,23]$. The temperature turns out to be coincide with the phase transition temperature predicted by the Lattice QCD calculations [23, 24]. More importantly, the non-zero collective velocity for heavy flavor and $J / \psi[25,26]$, shown in Fig. 1, is the first indication of light-flavor thermalization in central $A u+A u$ collisions at RHIC. 


\section{Summary}

In summary, the bulk matter created at RHIC demonstrates a strong collective expansion with large values of elliptic flow - the development of partonic collectivity. The heavy flavor hadrons like $D^{0}$ and $J / \psi$ also show non-zero collectivity in central $A u+A u$ collisions. The lower limit of the collective velocity is $\left\langle\beta_{T}\right\rangle_{p} \geq 0.2$ (c) at a temperature close to phase transition. These observations along with the 'jet-quenching', the multistrange hadrons $v_{2}[27,28]$ and the number of quark scaling in $v_{2}$ [7] point to the direction of thermlization for the matter produced at RHIC.

We thank the conference organizers. We are grateful for many enlightening discussions with Drs. P. Braun-Munzinger, S. Esumi, M. Gyulassy, U. Heinz, H. Huang, P. Huovinen, F. Liu, H.G. Ritter, K. Schweda, P. Sorensen, Z. Xu and Y.F. Zhang. This work was supported by the U.S. Department of Energy under Contract No. DE-AC0376SF00098.

[1] H. Satz, Nucl. Phys. A715, 3c(2003).

[2] F. Karsch, Nucl. Phys. A698, 199c(2002).

[3] Z. Fodor, Nucl. Phys. A715, 319c(2003) and references there in; Z. Fodor and S.D. Katz, Phys. Lett. B534, 87(2002).

[4] W. Reisdorf and H.G. Ritter, Ann. Rev. Nucl. Part. Sci. 47, 663(1997).

[5] E.L. Bratkovskaya, W. Cassing, H. Stoecker, and N. Xu, Phys. Rev. C71, 044901(2005) and references therein.

[6] P. Kolb and R. Rapp, Phys. Rev. C62, 054909(2000).

[7] Z. Lin and C. Ko, Phys. Rev. Lett. 89, 202302(2002); R.J. Fries, B. Mueller, C. Nonaka, S.A. Bass, Phys. Rev. Lett. 90, 202303(2003); D. Molnar and S. Voloshin, Phys. Rev. Lett. 91, 092301(2003).

[8] M. Gyulassy, I. Vitev, X.N. Wang, and B. Zhang, nucl-th/0302077 and references therein.

[9] J. Adams et al., (STAR Collaboration), Nucl. Phys. A757, 102(2005).

[10] N. Xu, Prog. Part. Nucl. Phys. 53, 165(2004).

[11] N. Xu, Nucl. Phys. A751, 109(2005).

[12] S.S. Adler et al., (PHENIX Collaboration), Phys. Rev. C69, 034909(2004).

[13] J. Adams et al., (STAR Collaboration), Phys. Lett. 612, 181(2005).

[14] J. Adams et al., (STAR Collaboration), Phys. Rev. Lett. 92, 112301(2004).

[15] K. Schweda et al., (STAR Collaboration), J. Phys. G: Nucl. Part. Phys., 30, S693(2004); J. Adams, nucl-exp/0606014.

[16] N. Xu and K. Masashi, Nucl. Phys. A698, 306c(2001).

[17] S.V. Afanasiev et al., (NA49 Collaboration), Phys. Rev. C66, 054902 (2002).

[18] H. Buesching and T. Gunji, QM05 conference, August, 2005.

[19] I. G. Bearden et al., (NA44 Collaboration), Phys. Rev. Lett 78, 2080(1997).

[20] H. van Hecke, H. Sorge, and N. Xu, Phys. Rev. Lett., 81, 5764(1998).

[21] Y. Cheng, F. Liu, Z. Liu, K. Schweda, and N. Xu, Phys. Rev. C68, 034901(2003).

[22] E. Schnedermann, J. Sollfrank, and U. Heinz, Phys. Rev. C48, 2462(1993).

[23] P. Braun-Munzinger, these proceedings.

[24] J. Cleymans, these proceedings.

[25] Y.F. Zhang, these proceedings.

[26] S. Esumi, these proceedings.

[27] S. Blyth, these proceedings.

[28] M. Oldenburg, (STAR Collaboration), nucl-ex/0510026. 